\title{
Performance evaluation of WMN-GA for different mutation and crossover rates considering number of covered users parameter
}

\author{
Tetsuya Oda ${ }^{\mathrm{a}, *}$, Admir Barolli ${ }^{\mathrm{b}}$, Fatos Xhafa ${ }^{\mathrm{c}}$, Leonard Barolli ${ }^{\mathrm{d}}$, Makoto Ikeda ${ }^{\mathrm{d}}$ and \\ Makoto Takizawa ${ }^{\mathrm{b}}$ \\ ${ }^{a}$ Graduate School of Engineering, Fukuoka Institute of Technology (FIT), Higashi-Ku, Fukuoka, Japan \\ ${ }^{\mathrm{b}}$ Department of Computers and Information Science, Seikei University, Musashino-Shi, Tokyo, Japan \\ ${ }^{\mathrm{c}}$ Department of Languages and Informatics Systems, Technical University of Catalonia, Barcelona, \\ Spain \\ ${ }^{\mathrm{d}}$ Department of Information and Communication Engineering, Fukuoka Institute of Technology (FIT), \\ Higashi-Ku, Fukuoka, Japan
}

\begin{abstract}
Node placement problems have been long investigated in the optimization field due to numerous applications in location science and classification. Facility location problems are showing their usefulness to communication networks, and more especially from Wireless Mesh Networks (WMNs) field. Recently, such problems are showing their usefulness to communication networks, where facilities could be servers or routers offering connectivity services to clients. In this paper, we deal with the effect of mutation and crossover operators in GA for node placement problem. We evaluate the performance of the proposed system using different selection operators and different distributions of router nodes considering number of covered users parameter. The simulation results show that for Linear and Exponential ranking methods, the system has a good performance for all rates of crossover and mutation.
\end{abstract}

\section{Introduction}

With the emergence of several new networking paradigms such as Ad-hoc networks, sensor networks, vehicular networks, cellular networks, optimization modelling and resolution turns out to be crucial to achieve optimized performance networks [1-3]. One such networking paradigm that is requiring the resolution of optimization problems is that of WMNs. At the heart of WMNs are the issues of achieving network connectivity and stability as well as QoS in terms of user coverage. These issues are very closely related to the family of node placement problems in WMNs, such as mesh router nodes placement. Node placement problems have been long investigated in the optimization field due to numerous applications in location science and classification (clustering). In such problems, we are given a number of potential facilities to serve to costumers connected to facilities aiming to find locations such that the cost of serving

\footnotetext{
${ }^{*}$ Corresponding author. E-mail: oda.tetuya.fit@gmail.com.
} 
to all customers is minimized. In traditional versions of the problem, facilities could be hospitals, polling centers, fire stations serving to a number of clients and aiming to minimize some distance function in a metric space between clients and such facilities.

WMNs $[4,5]$ infrastructures are currently used in developing and deploying medical, transport and surveillance applications in urban areas, metropolitan, neighboring communities and municipal area networks [6]. WMNs are based on mesh topology, in which every node (representing a server) is connected to one or more nodes, enabling thus the information transmission in more than one path. The path redundancy is a robust feature of this kind of topology. Compared to other topologies, mesh topology needs not a central node, allowing networks based on such topology to be self-healing. These characteristics of networks with mesh topology make them very reliable and robust networks to potential server node failures. In WMNs mesh routers provide network connectivity services to mesh client nodes. The good performance and operability of WMNs largely depends on placement of mesh routers nodes in the geographical deployment area to achieve network connectivity, stability and user coverage. The objective is to find an optimal and robust topology of the mesh router nodes to support connectivity services to clients.

For the mesh router node placement problem, are given a grid area arranged in cells where to distribute a number of mesh router nodes and a number of mesh client nodes of fixed positions (of an arbitrary distribution) in the grid area. The objective is to find a location assignment for the mesh routers to the cells of the grid area that maximizes the network connectivity and client coverage. Network connectivity is measured by the size of the giant component of the resulting WMN graph, while the user coverage is simply the number of mesh client nodes that fall within the radio coverage of at least one mesh router node.

For most formulations, node placement problems are shown to be computationally hard to solve to optimality [7-10], and therefore heuristic and meta-heuristic approaches are useful approaches to solve the problem for practical purposes. Several heuristic approaches are found in the literature for node placement problems in WMNs [11-15].

Genetic Algorithms (GAs) have been recently investigated as effective resolution methods. In this work, we present the results of the effect of mutation and crossover operators in GA for mesh router nodes placement problem. The study aims to identify the mutation and crossover types that work best for instances of different characteristics. A bi-objective optimization, namely, the maximization of the size of the giant component in the network (for measuring network connectivity) and that of user coverage are considered. However in this paper, we use only the number of covered users as a parameter for the system evaluation. In the simulations, we have used different distributions of mesh node clients (Uniform, Normal, Exponential and Weibull).

The rest of the paper is organized as follows. In Section 2 is presented Genetic Algorithm template and its application to mesh router nodes placement. We focus further on mutation types and mutation rate values in Section 3. The crossover operator is described in Section 4. The simulation results are given in Section 5. We end the paper in Section 6 with some conclusions.

\section{Genetic algorithms}

Genetic Algorithms (GAs) [16,17] have shown their usefulness for the resolution of many computationally combinatorial optimization problems. For the purpose of this work, we have used the template given in Algorithm 1.

We present next the particularization of GAs for the mesh router nodes placement in WMNs (see [20] for more details). 


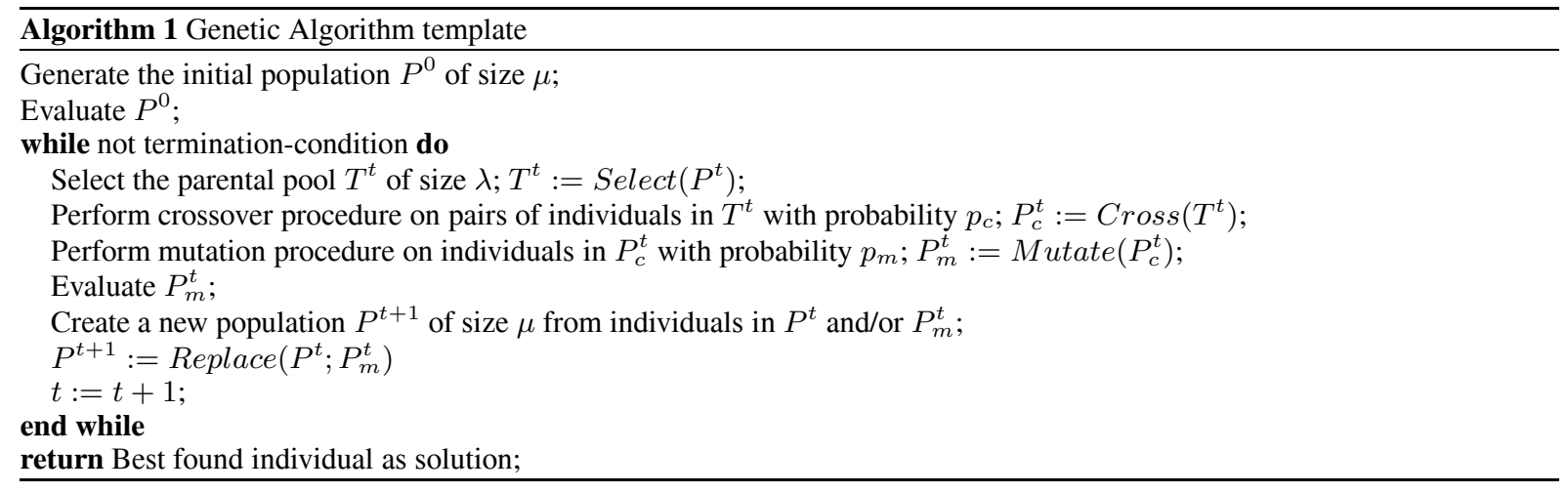

\subsection{Encoding}

The encoding of individuals (also known as chromosome encoding) is fundamental to the implementation of GAs in order to efficiently transmit the genetic information from parents to offsprings.

In the case of the mesh router nodes placement problem, a solution (individual of the population) contains the information on the current location of routers in the grid area as well as information on links to other mesh router nodes and mesh client nodes. This information is kept in data structures, namely, pos_routers for positions of mesh router nodes, routers_links for link information among routers and client_router_link for link information among routers and clients (matrices of the same size as the grid area are used.) Based on these data structures, the size of the giant component and the number of users covered are computed for the solution.

It should be also noted that routers are assumed to have different radio coverage, therefore any router could be linked to a number of clients and other routers. Obviously, whenever a router is moved to another cell of the grid area, the information on links to both other routers and clients must be computed again and links are re-established.

\subsection{Selection operators}

In the evolutionary computing literature we can find a variety of selection operators, which are in charge of selecting individuals for the pool mate. The operators considered in this work are those based on Implicit Fitness Re-mapping technique. It should be noted that selection operators are generic ones and do not depend on the encoding of individuals.

- Random Selection: This operator chooses the individuals uniformly at random. The problem is that a simple strategy does not consider even the fitness value of individuals and this may lead to a slow convergence of the algorithm.

- Best Selection: This operator selects the individuals in the population having higher fitness value. The main drawback of this operator is that by always choosing the best fitted individuals of the population, the GA converges prematurely.

- Linear Ranking Selection: This operator follows the strategy of selecting the individuals in the population with a probability directly proportional to its fitness value. This operator clearly benefits the selection of best endowed individuals, which have larger chances of being selected.

- Exponential Ranking Selection: This operator is similar to Linear Ranking but now probabilities of ranked individuals are weighted according to an exponential distribution. 
- Tournament Selection: This operator selects the individuals based on the result of a tournament among individuals. Usually winning solutions are the ones of better fitness value but individuals of worse fitness value could be chosen as well, contributing thus to avoiding premature convergence. Particular cases of this operator are the Binary Tournament and $N$-Tournament Selection, for different values of $N$.

\subsection{Crossover operators}

The crossover operators are the most important ingredient of GAs. Indeed, by selecting individuals from the parental generation and interchanging their genes, new individuals (descendants) are obtained. It is very important to stress that the crossover operators depend on the chromosome representation. The crossover operator should thus take into account the specifics of mesh router nodes encoding.

\subsection{Mutation operators}

Mutation operator is one of the GA ingredients. Unlike crossover operators, which achieve to transmit genetic information from parents to offsprings, mutation operators usually make some small local perturbation of the individuals, having thus less impact on newly generated individuals.

Crossover is "a must" operator in GA and is usually applied with high probability, while mutation operators when implemented are applied with small probability. The rationale is that a large mutation rate would make the GA search to resemble a random search. Due to this, mutation operator is usually considered as a secondary operator.

\section{Mutation in genetic algorithms}

\subsection{Mutation operators issues}

Many studies in the literature have shown that mutation when effectively combined with selection operators can improve the performance of GAs. Some of the important issues behind the use of mutation operators are as follows:

- The design of different versions of mutation operators and their evaluation in GA in conjunction with selection operators: In general, it is difficult to know a priori if a simple mutation, such as bit-flip mutation, would produce desired effect. Therefore different types of mutation operators should be designed and evaluated. In fact, their evaluation should be done in conjunction with selection operators.

- Mutation Rate: This is among most critical aspects in applying mutation operators. In most applications, mutation rate is kept low and constant through the search. However, the concrete mutation rate has to be tuned. In some works the use of dynamic mutation rates have also been proposed.

- Effect of Mutation Rate on GA Evolution: In GAs one critical aspect is keeping diversity of the population and avoid premature convergence to local optima. Certainly, mutation, through small perturbation of the individuals can contribute to maintaining the diversity of the population, if appropriate mutation rates are achieved. 
- Generic vs. Ad Hoc Problem-oriented Mutation Operators: In general, one could consider the mutation operator as generic, problem-independent operator. This is so because in most cases, small local perturbation to individuals such as bit flip, swap, and so on can be implemented similarly for most combinatorial structures. However, it might be interesting to explore the characteristics of the problem at hand and design some ad hoc problem-dependent operators.

\subsection{Setting mutation rates}

Setting the values of parameters in GAs has been and is still a major issue in GA research field. One such parameter is the mutation rate. The complexity of tuning GA parameters, and the mutation rate in particular, comes from the side-effect of one operator on the rest of operators. Ideally, tuning should be able to explore the synergies among different operators.

\subsubsection{Population size, crossover and mutation rate}

Among the different GA parameters, population size, crossover rate and mutation rate have considerable effect on GA convergence and, consequently, the quality of solution found. Some research studies reported in the literature suggest that there are direct relations among selection strategies, crossover rate and mutation rate. Regarding population size vs. mutation rate, the issue here is whether it would be more beneficial for the genetic search to use: (a) a small population size and rather large mutation rate, or (b) large population sizes and low mutation rate. The choice could have a significant impact on the GA convergence.

\subsubsection{Mutation rate}

As can be seen from above, setting the mutation value is difficult, in part due to the many other GA parameters to be set and the existing synergies among them. In the literature we would find essentially two ways to set the mutation rate: (a) using constant values and (b) using variable mutation rate. In the former, considered as the easiest way, the mutation rate is kept unchanged during the whole genetic search process. In this case, the value of the mutation rate can be fixed either a priori (based on an experimental study) or computed based on some problem size parameter. For instance, some authors have explored the advantages and limitations of using the $1 / L$ heuristic, in which the mutation rate is set to $1 / L$, where $L$ stands for the chromosome string length [18].

In the second group, mutation rate is changed during the genetic search process. The difficulty here is how to find an appropriate decreasing function of mutation rate. One such possible function is linear function, that is, mutation rate linearly decreases along the search process. One implementation would then be to keep mutation rate constant during a certain number of generations and then decrease it linearly. Some more advanced schemes proposed in the literature suggest using self-adaptive mutation rates either by splitting the population into sub-populations and using specific mutation rates to sub-populations or by using a ranking of the individuals in the population and then assign to individuals mutation according to the rank [19].

\subsection{Mutation operators for mesh routers nodes placement in WMNs}

In the case of mesh routers node placement, the matrix representation is chosen for the individuals of the population, in order to keep the information on mesh router nodes positions, mesh client positions, links among routers and links among routers and clients. The definition of the mutation operators is therefore specific to matrix-based encoding of the individuals of the population. Several specific mutation operators were considered in this study, which are move-based and swap-based operators. 


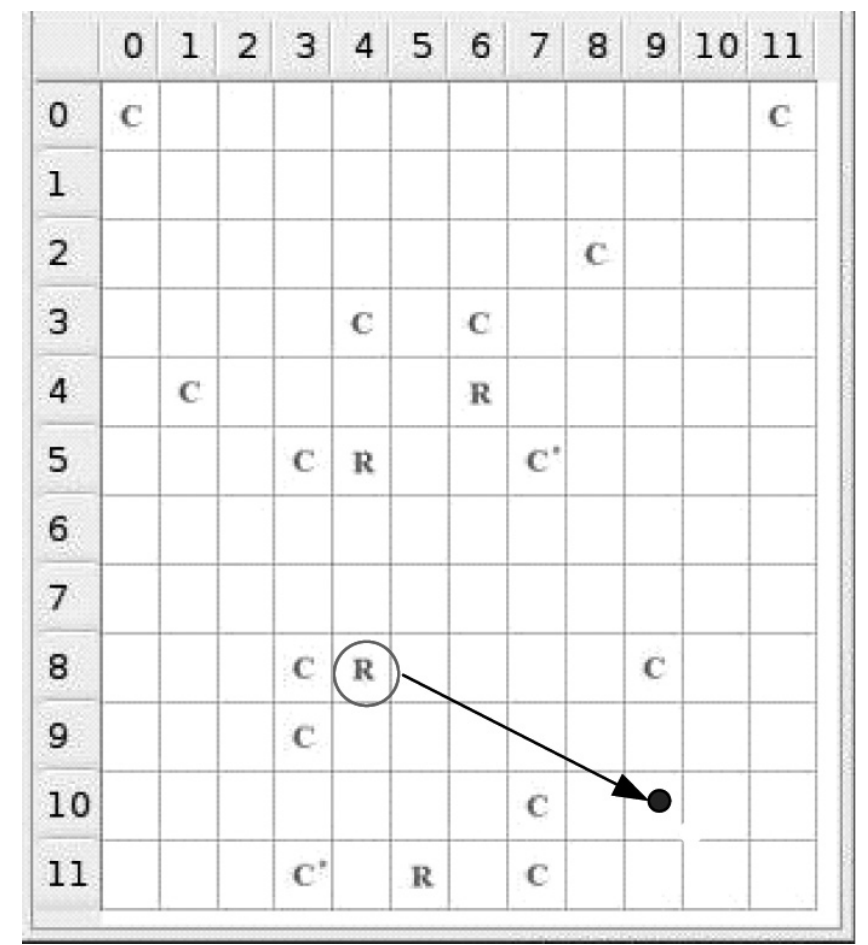

Fig. 1. Single mutate operator.

\subsubsection{SingleMutate}

This is a move-based operator. It selects a mesh router node in the grid area and moves it to another cell of the grid area (see Fig. 1).

\subsubsection{RectangleMutate}

This is a swap-based operator. In this version, the operator selects two "small" rectangles at random in the grid area, and swaps the mesh routers nodes in them (see Fig. 2).

\subsubsection{SmallMutate}

This is a move-based operator. In this case, the operator chooses randomly a router and moves it a small (a priori fixed) number of cells in one of the four directions: up, down, left or right in the grid (see Fig. 3). This operator could be used a number of times to achieve the effect of SingleMutate operator.

\subsubsection{SmallRectangleMutate}

This is a move-based operator. The operator selects first at random a rectangle and then all routers inside the rectangle are moved with a small (a priori fixed) numbers of cells in one of the four directions: up, down, left or right in the grid (see Fig. 4).

\subsubsection{Time efficiency of mutation and selection operators}

Simple mutation operators, such as bit-flip or simple move are very efficient as only small changes are done in the combinatorial structure. However, mutation based on making larger perturbations of the individual could be time costly given they are applied a total expected number of $p_{m} \cdot$ population_size, 


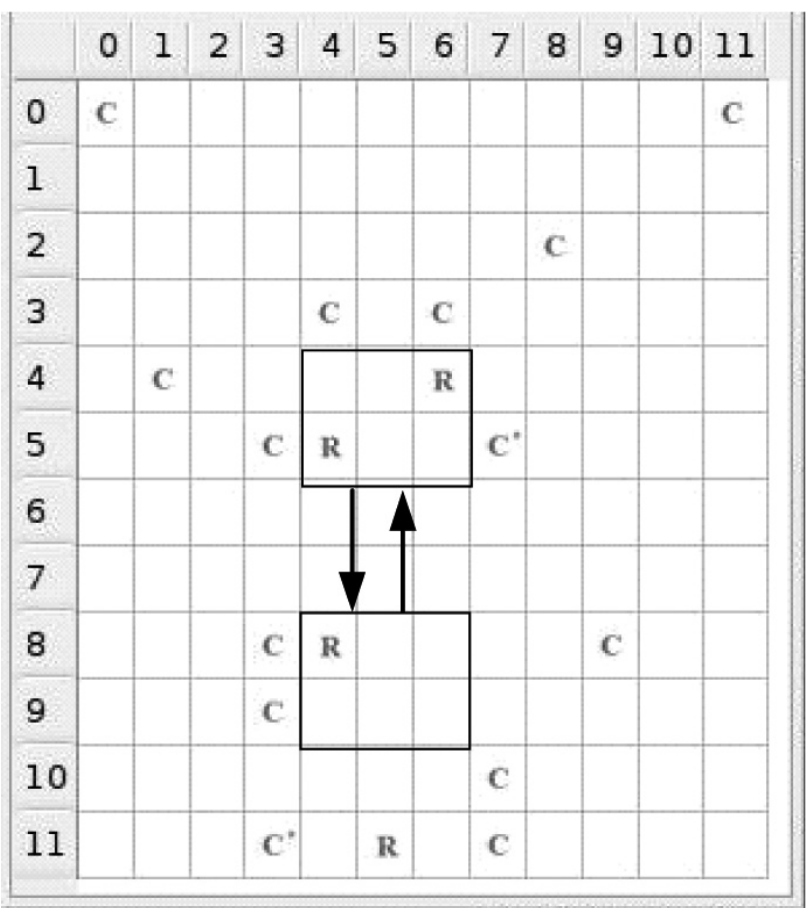

Fig. 2. Rectangle mutate operator.

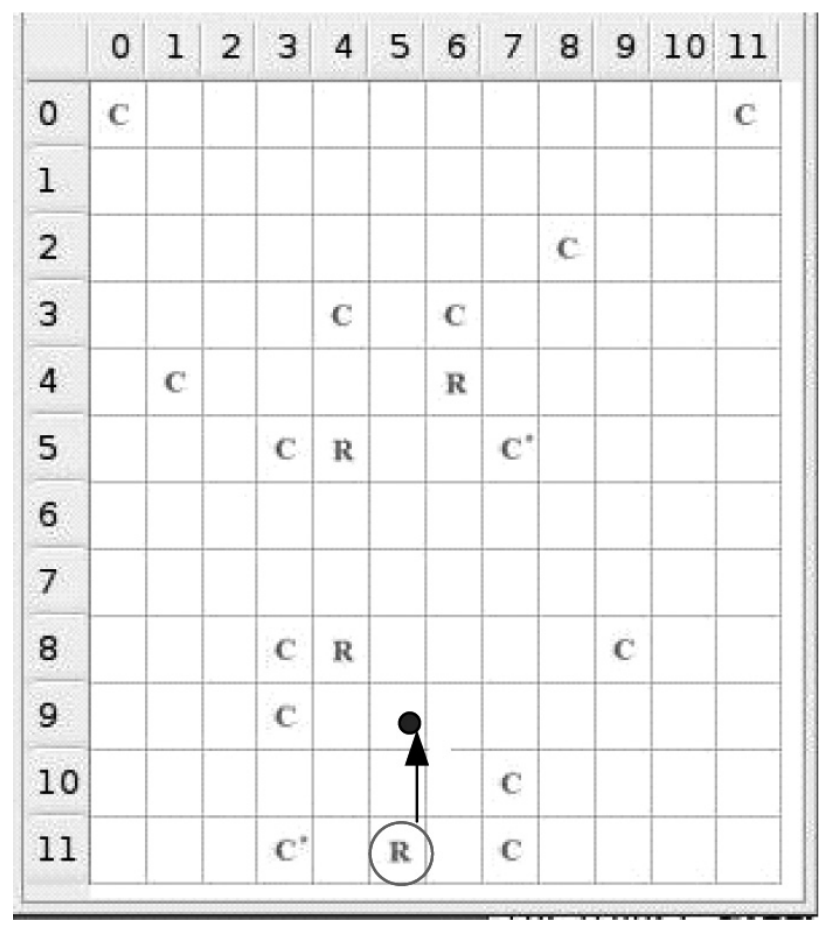

Fig. 3. Small mutate operator. 


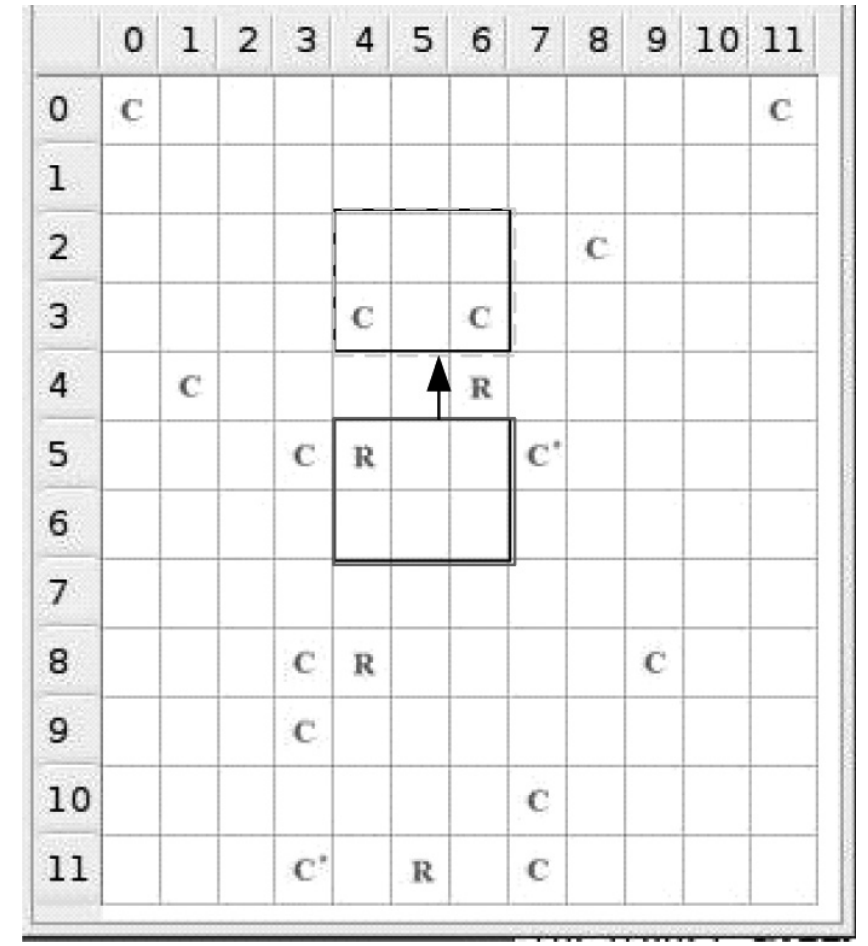

Fig. 4. Small rectangle mutate operator.

where $p_{m}$ denotes the mutation probability. This amount is to be multiplied by the time cost of performing one mutation, which in case of rectangle mutate and small rectangle mutate are $O$ (grid_side $\times$ grid_side). The computational effort is even larger for the crossover operators as in this case the grid area has to be explored to find the sparsest and densest areas.

\subsection{Crossover operators}

The crossover operator selects individuals from the parental generation and interchanging their genes, thus new individuals (descendants) are obtained. The aim is to obtain descendants of better quality that will feed the next generation and enable the search to explore new regions of solution space not explored yet.

There exist many types of crossover operators explored in the evolutionary computing literature. It is very important to stress that crossover operators depend on the chromosome representation. This observation is especially important for the mesh router nodes problem, since in our case, instead of having strings we have a grid of nodes located in a certain positions. The crossover operator should thus take into account the specifics of mesh router nodes encoding. We have considered the following crossover operator, called intersection operator (denoted CrossRegion, hereafter), which take in input two individuals and produce in output two new individuals (see Algorithm 2).

\section{Proposed and implemented WMN-GA system}

In this section, we present WMN-GA system. Our system can generate instances of the problem using different distributions of client and mesh routers. 

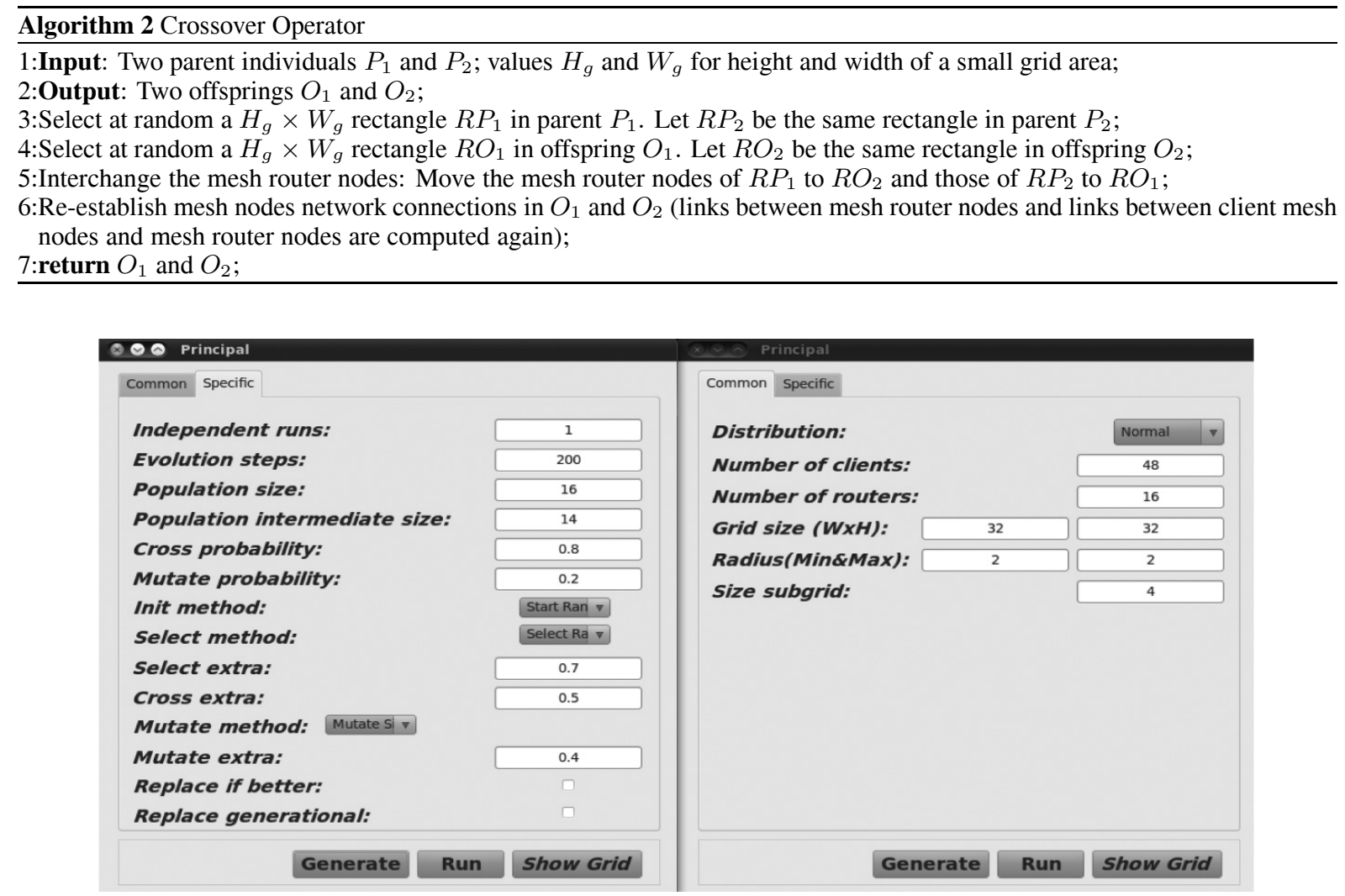

Fig. 5. GUI tool for WMN-GA system.

The GUI interface of WMN-GA is shown in Fig. 5. The left side of the interface shows the GA parameters configuration and on the right side are shown the network configuration parameters.

For the network configuration, we use: distribution, number of clients, number of mesh routers, grid size, radious of transmission distance and the size of subgrid.

For the GA parameter configuration, we use: number of independent runs, GA evalution steps, population size, population intermediate size, crossover probability, mutation probability, initial methods, select method.

\section{Simulation results}

We carried out many simulations to evaluate the performance of WMNs using WMN-GA system.

In this work, we considered the number of covered users parameter. The number of Mesh Routers is considered 16. We take in consideration four distribution methods: Exponetial, Normal, Uniform and Weibull. Six selection operators are used: Best, Exponential Ranking, Linear Ranking, Binary Tournament, N Tournament and Random.

In Figs 6, 7, 8 and 9 are used box plots to analyse the range of data values. Each box plot shows the median of each simulation data (the median is noted by the notch), the upper quartile ( 25 th and 75 th percentile, respectively) and the outliers. The whiskers rapresent the lowest data which is still within 


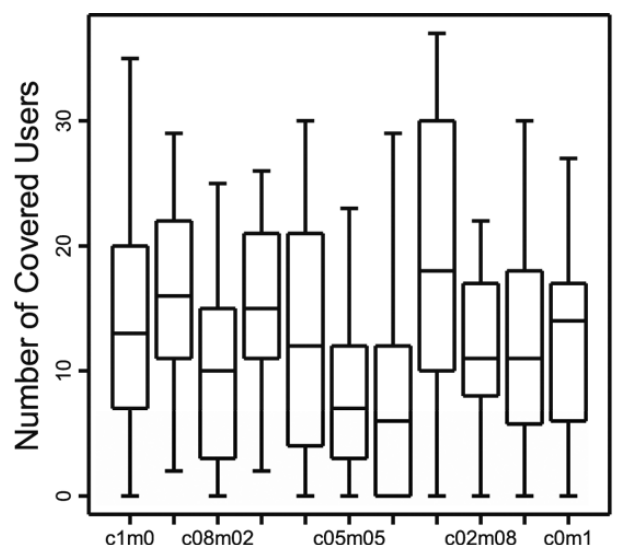

Ratio of Crossover and Mutation

(a) Best

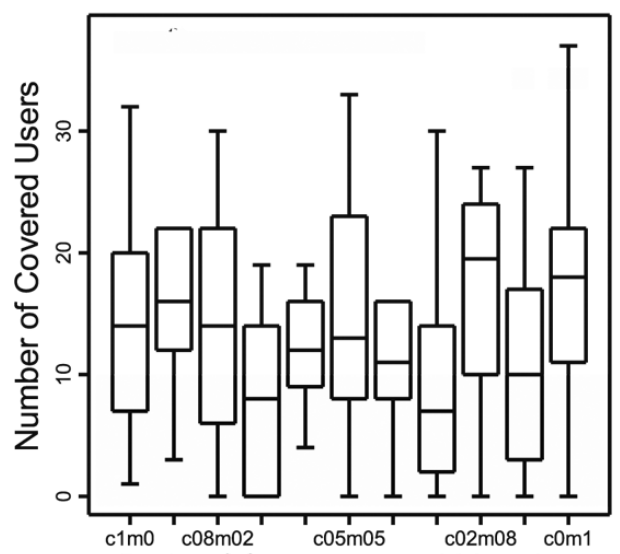

Ratio of Crossover and Mutation

(c) Linear Ranking

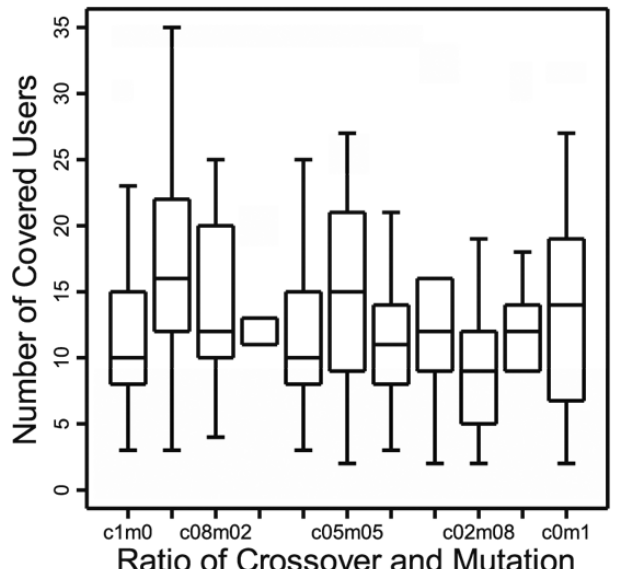

(e) N Tournament

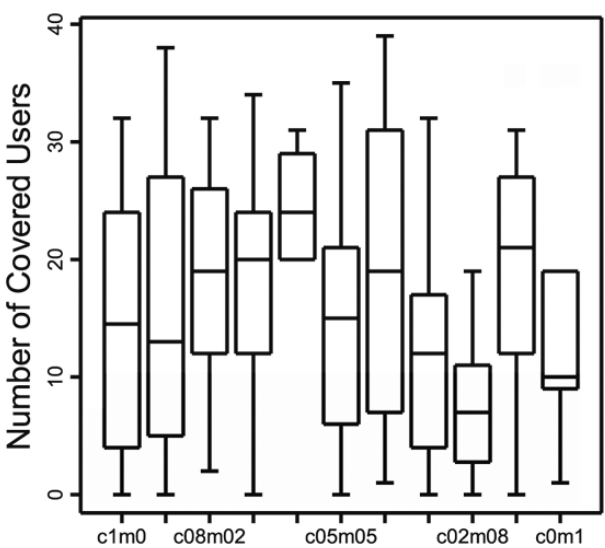

Ratio of Crossover and Mutation

(b) Exponential Ranking

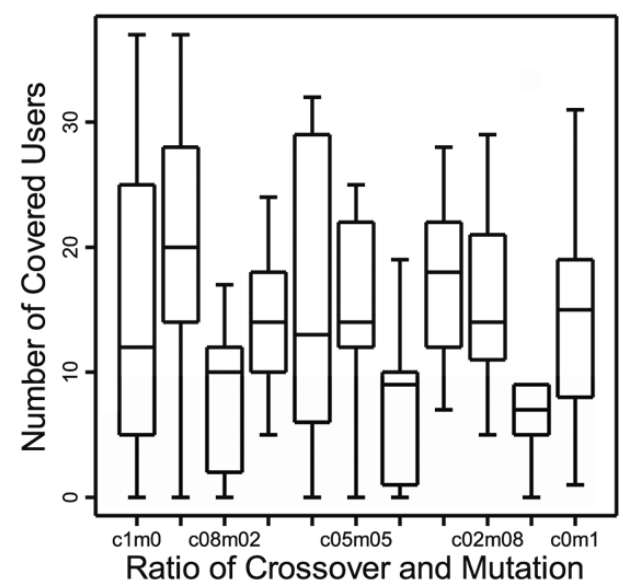

(d) Binary Tournament

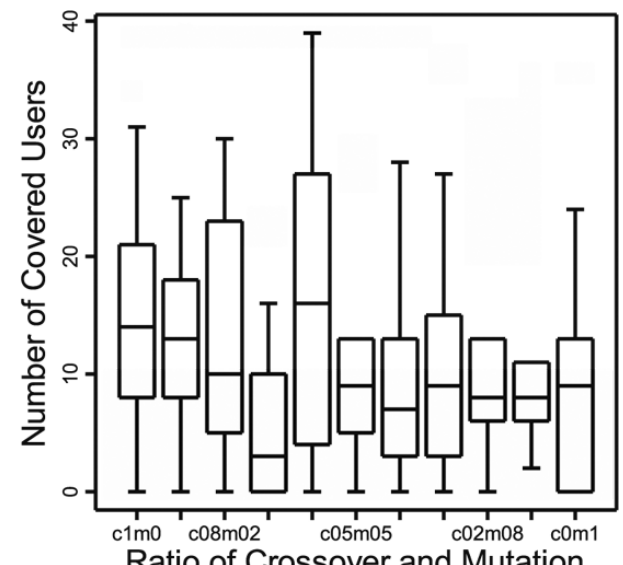

(f) Random

Fig. 6. Number of covered users vs. ratio of crossover and mutation for exponential distribution. 


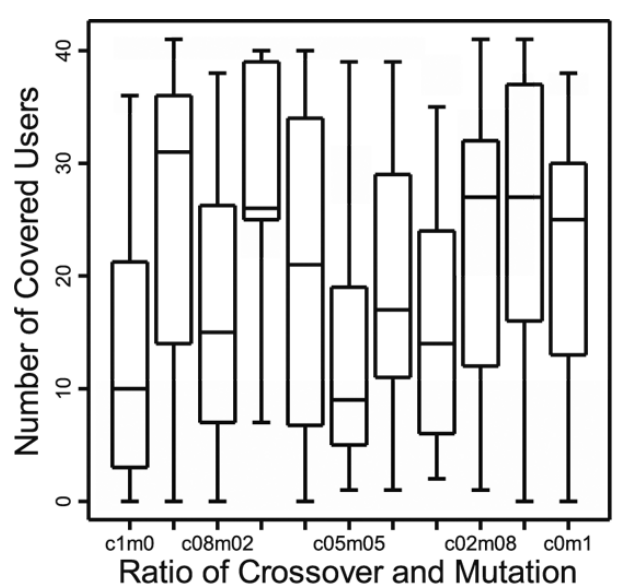

(a) Best

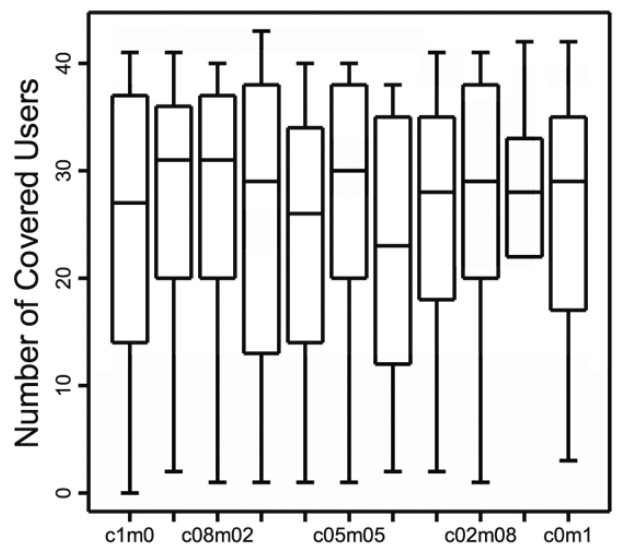

Ratio of Crossover and Mutation

(c) Linear Ranking

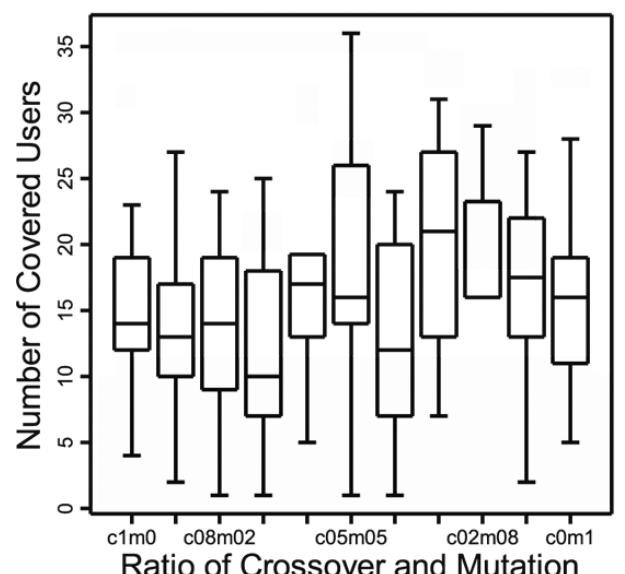

(e) N Tournament

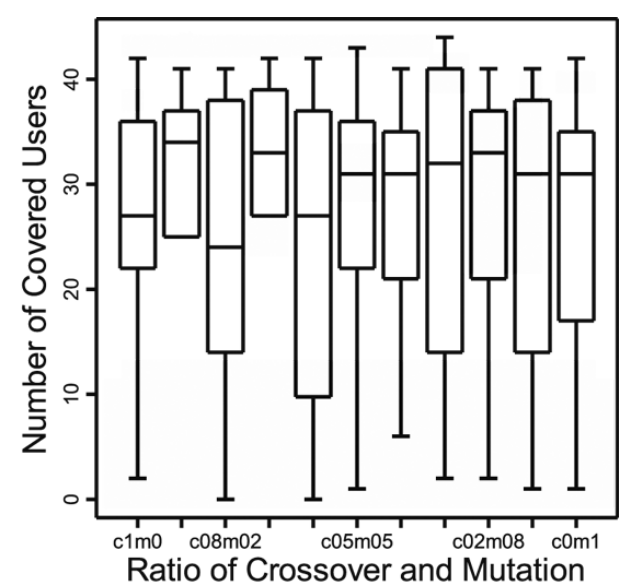

(b) Exponential Ranking

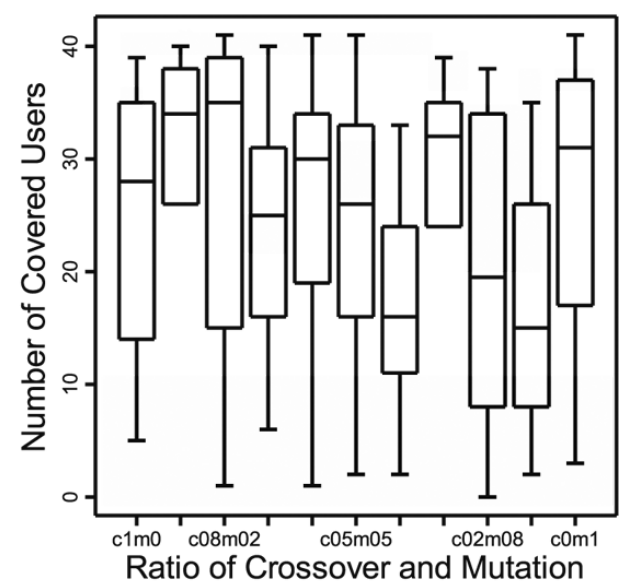

(d) Binary Tournament

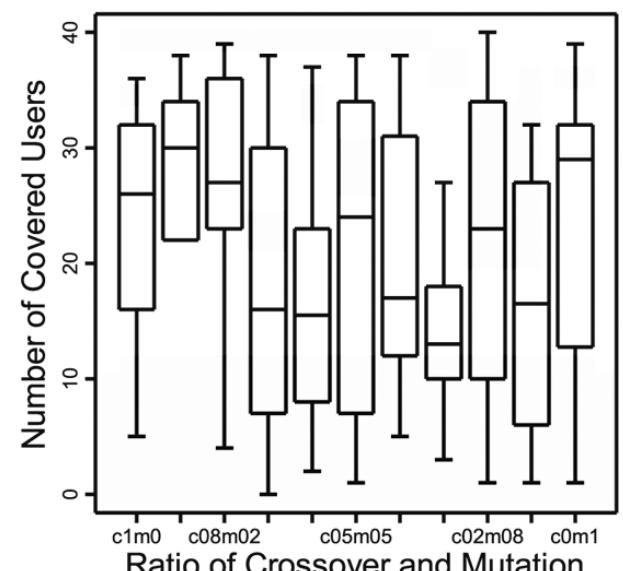

(f) Random

Fig. 7. Number of covered users vs. ratio of crossover and mutation for normal distribution. 


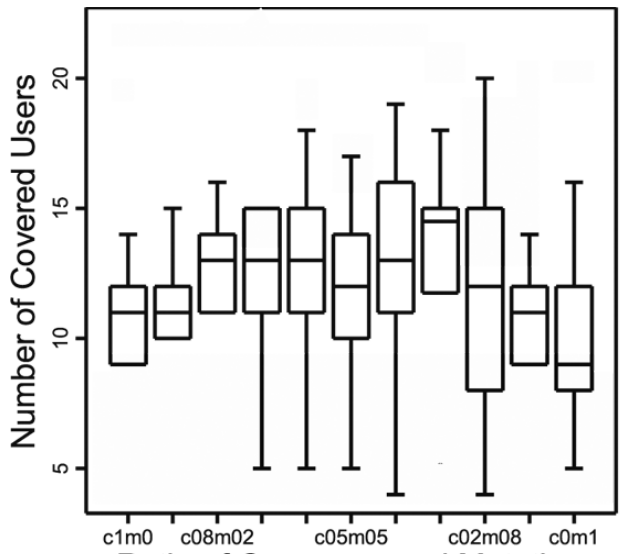

Ratio of Crossover and Mutation

(a) Best

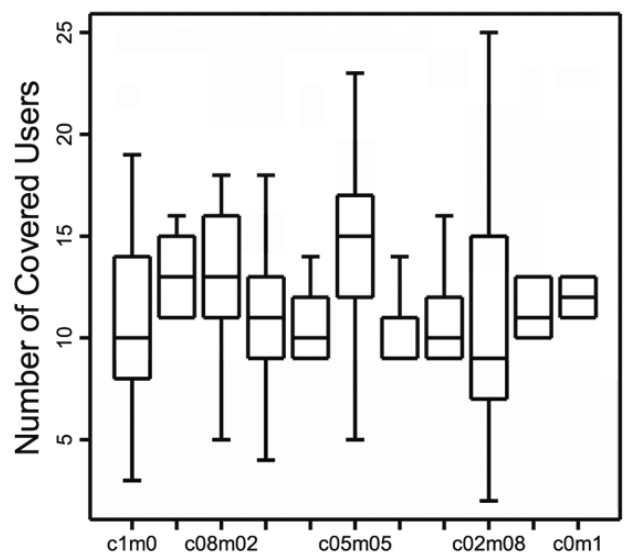

Ratio of Crossover and Mutation

(c) Linear Ranking

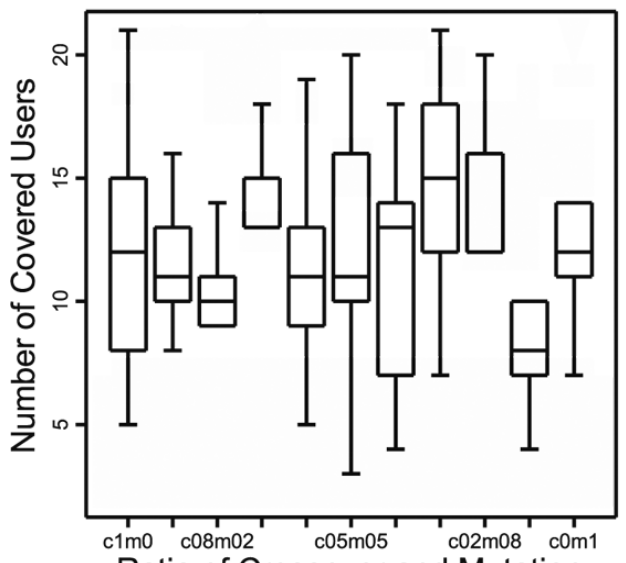

(e) N Tournament

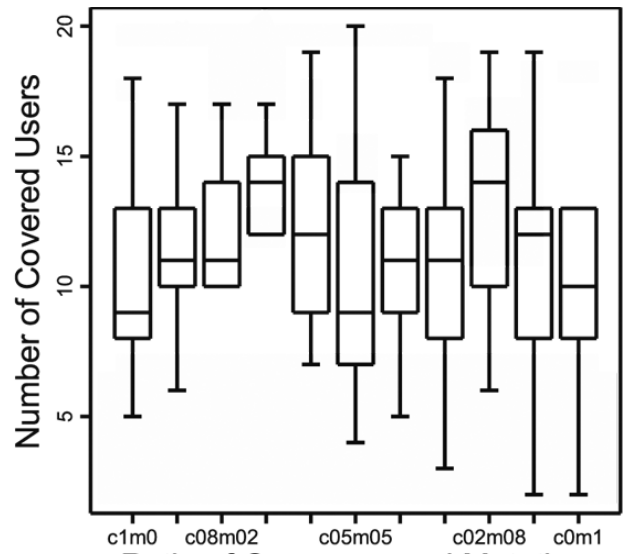

Ratio of Crossover and Mutation

(b) Exponential Ranking

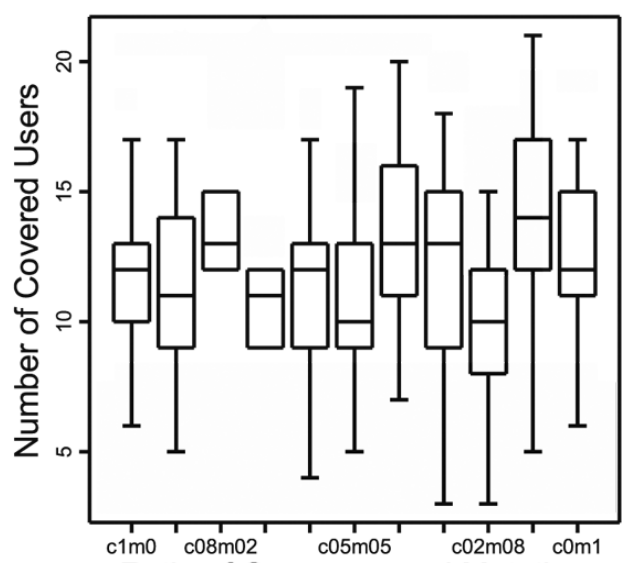

Ratio of Crossover and Mutation

(d) Binary Tournament

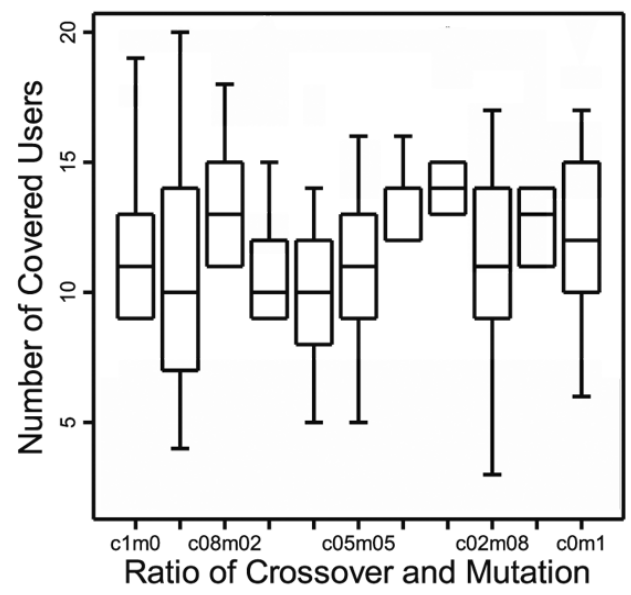

(f) Random

Fig. 8. Number of covered users vs. ratio of crossover and mutation for uniform distribution. 


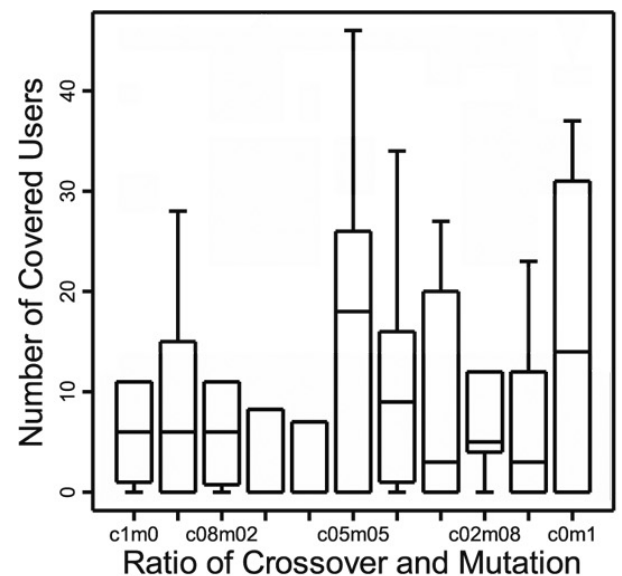

(a) Best

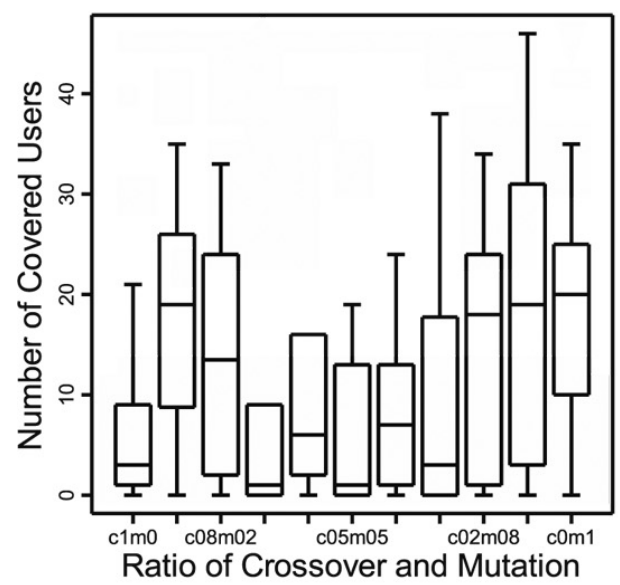

(c) Linear Ranking

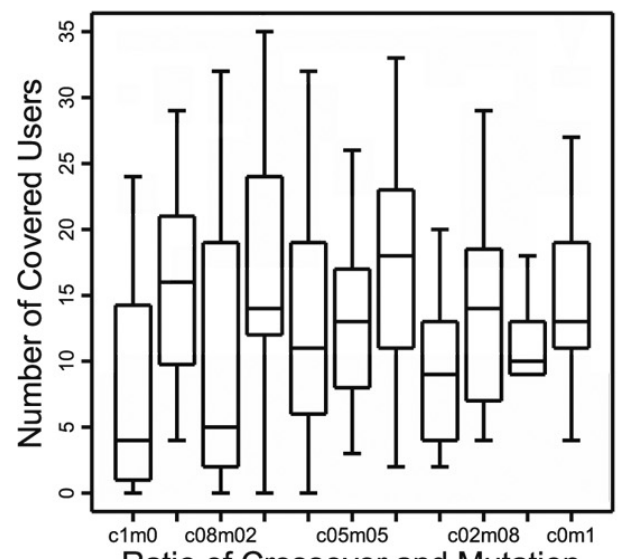

(e) N Tournament

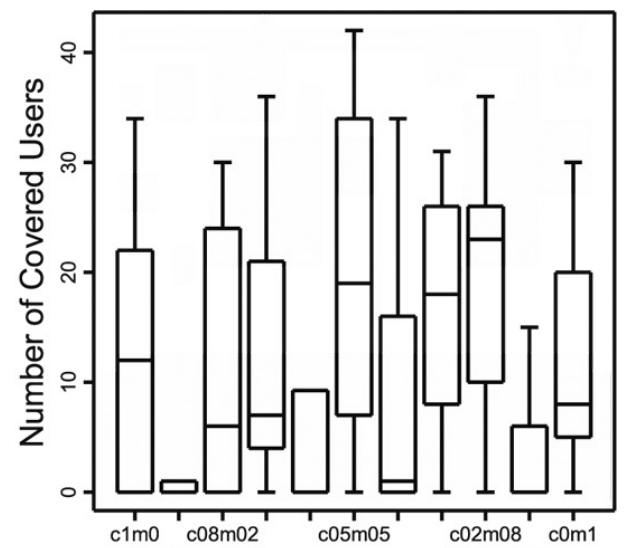

Ratio of Crossover and Mutation

(b) Exponential Ranking

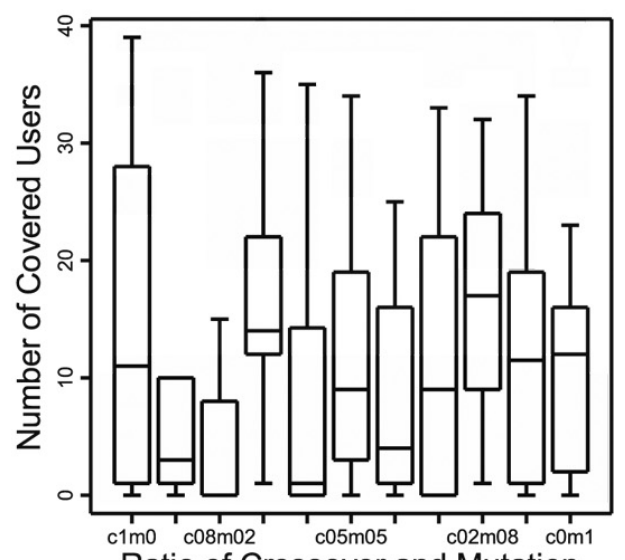

Ratio of Crossover and Mutation

(d) Binary Tournament

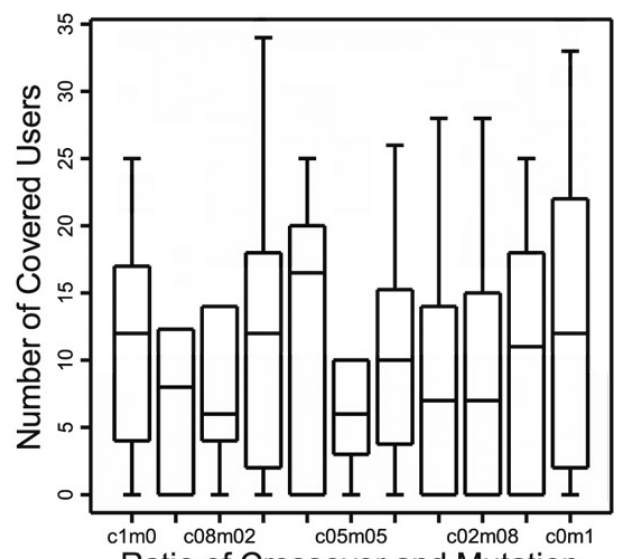

Ratio of Crossover and Mutation

(f) Random

Fig. 9. Number of covered users vs. ratio of crossover and mutation for weibull distribution. 
1.5 inter-quartile range of the lowest quartile and the highest data which is still within 1.5 inter-quartile range of the upper quartile.

In Fig. 6 is shown the number of covered users vs. ratio of crossover and mutation for exponential distribution. The maximal number of covered users among these selection operators is achieved by Exponential Ranking operator. The maximum number of covered users is 25 . From the results, we can see that Exponential Ranking has better performance. Also, Linear Ranking and Binary Tournament have better behaviour than Best, N Tournament and Random selection operators.

In Fig. 7 is shown the number of covered users vs. the ratio of crossover and mutation for normal distribution. From the results, we can see that Exponential Ranking and Linear Ranking operators have almost the same performance, the number of covered users is almost stable for different rates of crossover and mutation, but for $c=90 \%$ and $m=10 \%$ the Exponential Ranking has a bigger number of covered users (35 users). Best operator reaches the best coverage for $c=90 \%$ and $m=10 \%$ but for the other rates it covers only few users. For the Binary Tournament the maximal number of covered users is the same with Exponential Ranking but in Exponential Ranking the number of covered users is more stable for all rates of crossover and mutation. $\mathrm{N}$ Tournament operator has a bad coverage, the number of covered users is very small. For Random operator the number of covered users is bigger for $c=90 \%$ and $m=10 \%$ but for other rates it covers only a small number of users.

In Fig. 8 is shown the number of covered users vs. the ratio of crossover and mutation for uniform distribution. As can be seen, the performance for all these selection operators is almost the same and it is not good because the number of the covered users is less than 15 users.

In Fig. 9 is shown the number of coverd users vs. the ratio of crossover and mutation for weibull distribution. The maximum number of covered users is 23 users and is achieved when $c=20 \%$ and $m=80 \%$. Other operators offer a small number of covered users.

From all simulation results, we can see that for normal distribution, different rates of crossover and mutation, and different selection operators, the best performance is for Exponential Ranking.

\section{Conclusions}

In this work, we have proposed and implemented a system based on GAs to solve the connectivity problem in WMNs. We call this system WMN-GA.

We evaluated the performance of the proposed system using different genetic operators and different distributions of router nodes considering number of covered users parameter.

The simulation results show that for Exponential and Linear Ranking methods, the system has a good performance.

In the future work, we would like to compare the performance of WMN-GA system with other optimization algorithms.

\section{References}

[1] E. Kulla, M. Hiyama, M. Ikeda, L. Barolli, V. Kolici and R. Miho, MANET Performance for Source and Destination Moving Scenarios Considering OLSR and AODV Protocols, Mobile Information Systems (MIS), IOS Press 6(4) (2010), $325-339$.

[2] A. Durresi, M. Durresi and L. Barolli, Secure Authentication in Heterogeneous Wireless Networks, Mobile Information Systems (MIS) 4(2) (2008), 119-130.

[3] L. Barolli, A Speed-Aware Handover System for Wireless Cellular Networks Based on Fuzzy Logic, Mobile Information Systems (MIS), IOS Press 4(1) (2008), 1-12. 
[4] I.F. Akyildiz, X. Wang and W. Wang, Wireless Mesh Networks: A Survey, Computer Networks 47(4) (2005), 445-487.

[5] N. Nandiraju, D. Nandiraju, L. Santhanama, B. He, J. Wang and D. Agrawal, Wireless mesh networks: Current challenges and future direction of web-in-the-sky, IEEE Wireless Communications, 2007, pp. 79-89.

[6] C. Chen and C. Chekuri, Urban Wireless Mesh Network Planning: The Case of Directional Antennas, Tech Report No. UIUCDCS-R-2007-2874, 2007.

[7] M.R. Garey and D.S. Johnson, Computers and Intractability -A Guide to the Theory of NP-Completeness, Freeman, San Francisco, 1979.

[8] A. Lim, B. Rodrigues, F. Wang and Zh. Xua, $k$-Center Problems with Minimum Coverage, Theoretical Computer Science 332(1-3) (2005), 1-17.

[9] E. Amaldi, A. Capone, M. Cesana, I. Filippini and F. Malucelli, Optimization Models and Methods for Planning Wireless Mesh Networks, Computer Networks 52 (2008), 2159-2171.

[10] J. Wang, B. Xie, K. Cai and D.P. Agrawal, Efficient Mesh Router Placement in Wireless Mesh Networks, MASS, Pisa, Italy, 2007, pp. 9-11.

[11] S.N. Muthaiah and C. Rosenberg, Single Gateway Placement in Wireless Mesh Networks, In Proc. of 8th International IEEE Symposium on Computer Networks, Turkey, 2008, pp. 4754-4759.

[12] P. Zhou, B.S. Manoj and R. Rao, A Gateway Placement Algorithm in Wireless Mesh Networks, In Proc. of the 3rd International Conference on Wireless Internet, Austin, Texas, 2007, pp. 1-9.

[13] M. Tang, Gateways Placement in Backbone Wireless Mesh Networks, International Journal of Communications, Network and System Sciences 2(1) (2009), 45-50.

[14] A. Antony Franklin and C. Siva Ram Murthy, Node Placement Algorithm for Deployment of Two-Tier Wireless Mesh Networks, In Proc. of IEEE GLOBECOM-2007, Washington, USA, 2007, pp. 4823-4827,

[15] T. Vanhatupa, M. Hännikäinen and T.D. Hämäläinen, Genetic Algorithm to Optimize Node Placement and Configuration for WLAN Planning, In Proc. of 4th International Symposium on Wireless Communication Systems, 2007, pp. $612-616$.

[16] J. Holland, Adaptation in Natural and Artifitial Systems, University of Michigan Press, Ann Arbor, 1975.

[17] A. Barolli, E. Spaho, L. Barolli, F. Xhafa and M. Takizawa, QoS Routing in Ad-hoc Networks Using GA and Multiobjective Optimization, Mobile Information Systems (MIS), IOS Press 7(3) (2011), 169-188.

[18] G. Ochoa, Setting The Mutation Rate: Scope And Limitations Of The 1/L Heuristic, In Proc. of the Genetic and Evolutionary Computation Conference (GECCO-2002), USA, 2002, pp. 495-502.

[19] J. Cervantes and C.R. Stephens, Limitations of Existing Mutation Rate Heuristics and How a Rank GA Overcomes Them, IEEE Transactions on Evolutionary Computation 13(2) (2009), 369-397.

[20] F. Xhafa, Ch. Sanchez and L. Barolli, Genetic Algorithms for Efficient Placement of Router Nodes in Wireless Mesh Networks, Proc. of AINA 2010, 2010, pp. 465-472.

Tetsuya Oda received BE in Information and Communication Engineering from Fukuoka Institute of Technology (FIT), Japan in 2010. Presently, he is a Master Student at Graduate School of Engineering, FIT, Japan. His current research interests include wireless networks, mesh networks, ad-hoc networks and sensor networks.

Admir Barolli was graduated from Agricultural University of Tirana, Albania. He received his Diploma Degree in April 2008. From October 2009 to June 2010, he was a Visiting Researcher at Curtin University of Technology, Australia. Presently, he is a Visiting Researcher at the Department of Computer and Information Science, Seikei University, Japan and working toward his $\mathrm{PhD}$ Degree. He has been working for many years in Agriculture Sectors in Albania. His research interest are in genetics, genetic algorithms, agricultural engineering, intelligent algorithms, climate change, global warming, computer networks, ad-hoc networks, mesh networks and P2P systems.

Fatos Xhafa joined the Department of Languages and Informatics Systems of the Technical University of Catalonia as an Assistant Professor in 1996 and is currently an Associate Professor and member of the ALB- COM Research Group of this department. His current research interests include parallel algorithms, combinatorial optimization, approximation and metaheuristics, distributed programming, grid and P2P computing. He has published in leading international journals and conferences and has served in the organizing committees of many conferences and workshops. He is also a member of the editorial board of several international journals.

Leonard Barolli is a Professor at the Department of Information and Communication Engineering, Fukuoka Institute of Technology (FIT), Japan. He received BE and PhD Degrees from Tirana University and Yamagata University in 1989 and 1997 , respectively. He has published about 350 papers in Journals, Books and International Conference. He has served as a Guest Editor for many Journals. He was PC Chair of IEEE AINA-2004 and ICPADS-2005. He was General Co-Chair of IEEE AINA-2006, AINA-2008 and AINA-2010. He is Steering Committee Chair of CISIS and BWCCA International Conferences. His research interests include, $\mathrm{P} 2 \mathrm{P}$, intelligent algorithms, ad-hoc and sensor networks. He is a member of IEEE, IEEE Computer Society, IPSJ and SOFT. 
Makoto Ikeda is an Assistant Professor, at Fukuoka Institute of Technology (FIT), Japan. He was an Assistant Research Fellow at the Center for Asian and Pacific Studies, Seikei University, Japan from April 2010 to March 2011. He received BE, MS and $\mathrm{PhD}$ degrees in Information and Communication Engineering, Fukuoka Institute of Technology (FIT), Japan, in 2005, 2007, and 2010, respectively. He was a Japan Society for the Promotion of Science (JSPS) Research Fellow from April 2008 to March 2010. Dr. Ikeda has widely published in peer reviewed international journals and international conferences proceedings. He has served as PC Members for many international conferences. He is a member of IEEE, ACM, IPSJ and IEICE. His research interests include wireless networks, mobile computing, high-speed networks, P2P systems, mobile ad-hoc networks, wireless sensor networks and vehicular networks.

Makoto Takizawa is a Professor at the Department of Computer and Information Science, Seikei University. He was a Professor and the Dean of the Graduate School of Science and Engineering, Tokyo Denki University. He was a Visiting Professor at GMD-IPSI, Keele University, and Xidian University. He was on the Board of Governors and a Golden Core member of IEEE CS and is a fellow of IPSJ. He received his DE in Computer Science from Tohoku University. He chaired many international conferences like IEEE ICDCS, ICPADS, and DEXA. He is the founder of IEEE AINA. His research interests include distributed systems and computer networks. 

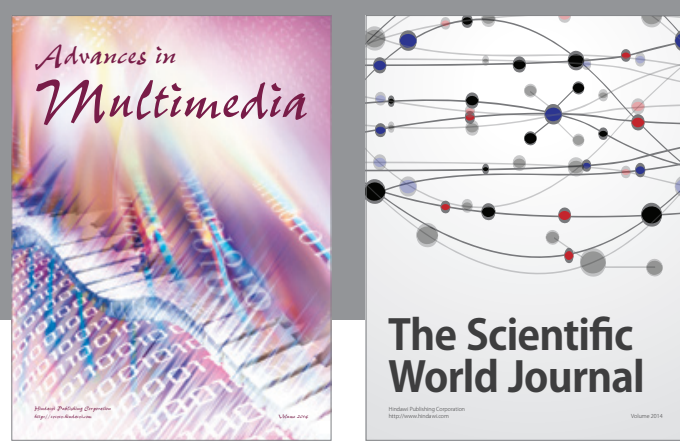

The Scientific World Journal
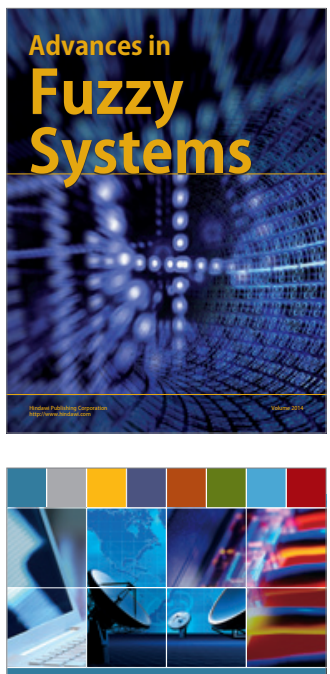

Computer Networks and Communications
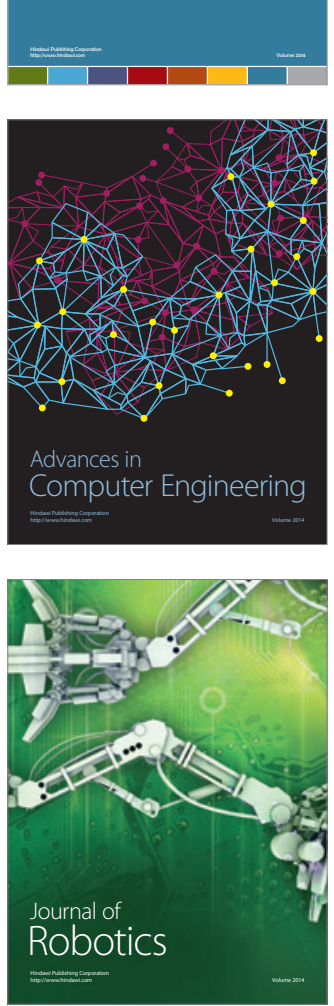
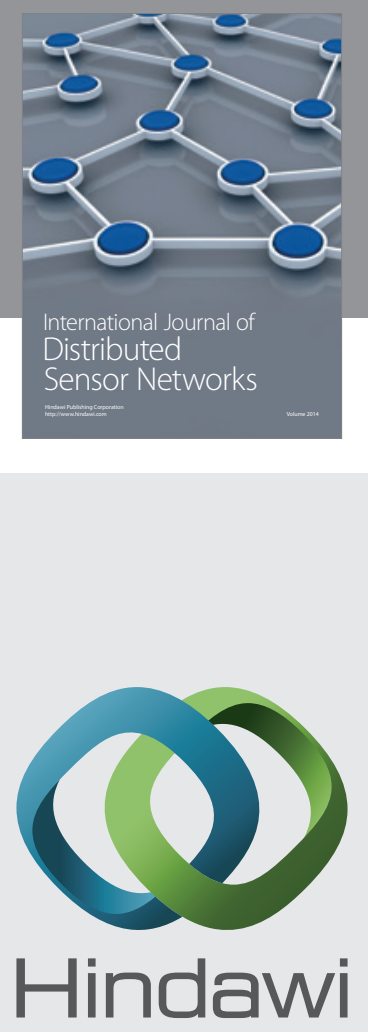

Submit your manuscripts at

http://www.hindawi.com
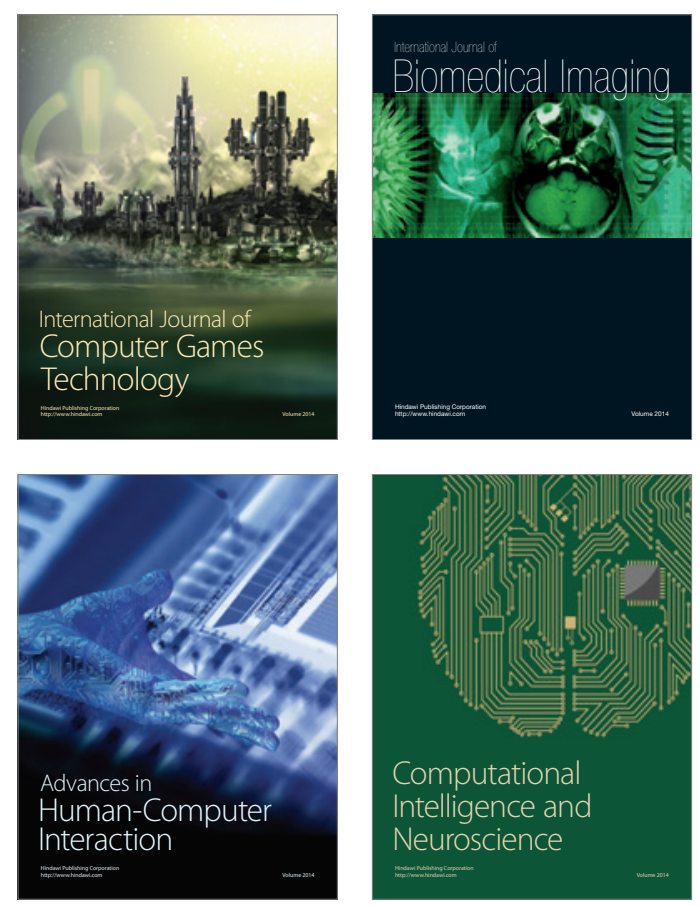
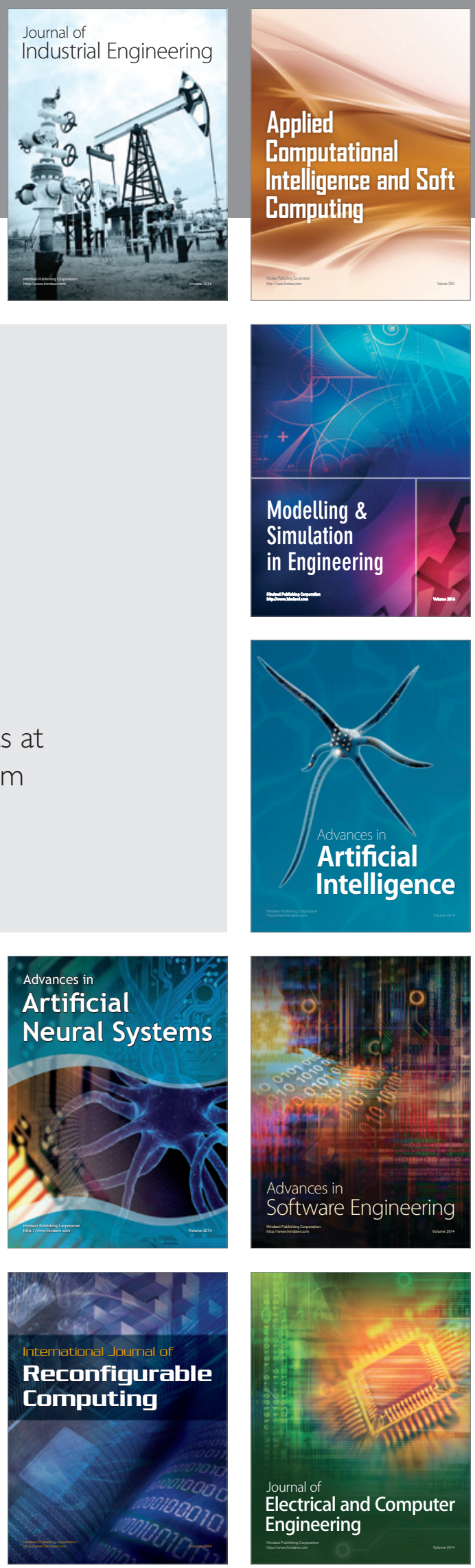